\title{
Study on Crisis Early-warning of Internet Rumors about Emergencies Based on BP Neural Network
}

Ying Zhou, Peng Zhang, Yuexin Lan, Haoqing Li

Chinese People's Armed Police Forces Academy, Langfang 065000, China

\section{基于 BP 神经网络的突发事件网络谣言危机预警 研究}

周颖, 张鹏, 兰月新, 李昊青

中国人民武装警察部队学院, 河北廊坊 065000 , 中国

\begin{abstract}
With the emergencies raising a media storm on the internet, it always brings all kinds of internet rumors at the same time, which not only damages the government's image, but also creates great obstacles to dealing with emergencies. Therefore, conducting risk assessments about Internet Rumors in emergencies is an effective measure to prevent and control Internet Rumors in public administration. In this paper, the BP neural network model is used for crisis warning of establishing an Internet rumors early-warning index system, and then the quantitative evaluation of risk for the Internet rumors can be achieved. We analyzed and verified the model through computer simulation experiments combined with the explosion event in Tianjin on August 12th, 2015. The conclusion suggests that it is feasible to use BP neural network model in crisis warning of Internet rumors, and the warning's grading result helps the government in preventing and controlling Internet rumors.
\end{abstract}

Key words: Internet Rumors, BP Neural Network, Crisis Warning, early warning indicator

\section{摘 要}

突发事件在网络上掀起與论风暴的同时, 往往会衍生各种各样的网络谣言, 不仅损害政 府形象, 也给突发事件处置带来了极大的阻碍, 因此对突发事件中的网络谣言进行风险评估 是公共管理中对网络谣言进行防治的有效举 措。本文在构建网络谣言预警指标体系的基础 上, 将 BP 神经网络模型用于网络谣言危机预 警, 进而实现突发事件网络谣言风险的定量评 判。最后通过计算机仿真实验, 结合 “8. 12 天津爆炸事件” 对该模型进行分析和验证。结 论表明, 将 BP 神经网络用于突发事件网络谣 言危机预警有较大的优势, 预警分级结果对于 政府进行网络谣言防治有一定的作用。

关键词: 网络谣言; BP 神经网络; 危机预警; 预警指标

1 引言

2016 年 1 月 22 日, 中国互联网络信息中 心在京发布第 37 次《中国互联网络发展状况 统计报告》。该报告显示, 截至 2015 年 12 月, 中国网民规模达 6.88 亿, 互联网普及率达到 


\section{Risk Analysis and Crisis Response in Big Data Era (RAC-16)}

$50.3 \%$, 中国居民上网人数已过半。报告显示, 我国手机网民规模达 6.20 亿, 手机成为拉动 网民规模增长的主要因素 ${ }^{[1]}$ 。迅速增长的网民 规模, 智能手机提供的随时随地的快捷上网方 式, 经济社会转型期积累的各种社会负面情绪, 为网络谣言滋生和蔓延提供了条件。网络谣言 由于其传播速度快, 波及范围广, 内容歪曲夸 大等特点，极容易引发社会恐慌，对正常的社 会秩序造成严重不良影响。

张鹏从网络谣言认知的角度, 采用Hayashi 数量化理论III的数值分析法实现了网络谣言 的数量化分类 ${ }^{[2]}$ 。敖炀借助奥尔伯特的谣言公 式, 提出网络谣言是属于网络群体性事件的一 个重要亚种, 谣言的产生和事件的重要性与模 糊性成正比, 事件越重要而且越模糊, 谣言传 播产生的效应也就越大 ${ }^{[3]}$ 。黄建提出网络谣言 的成因主要有: 社会利益格局的深刻调整是其 生发的首要根源; 公众责任感和判断力的不足 客观上助推了网络谣言的传播; 现有法制的规 则缺失使网络谣言有生存发展的空间; 截控谣 言的网络技术创新能力不足, 致使网络谣言得 以登堂入室 ${ }^{[4]}$ 。上述学者分别从网络谣言的分 类、传播机制和成因等方面对网络谣言进行了 研究, 但是目前对网络谣言预警的研究比较缺 乏, 大多集中在定性分析上, 缺乏定量的预警 研究。丁菊玲、薛圈圈等人也对 $B P$ 神经网络预 警方法进行了相关的研究, 但其研究方向主要 是网络與情危机预警。

本文在前人研究的基础上, 从网络谣言本 身的特征出发, 建立了网络谣言预警指标体系, 并通过构建基于 BP 神经网络的数学模型, 实 现对网络谣言的定量预警。

\section{2 网络谣言预警指标}

网络谣言可以看作信息异化的网络與情, 因此, 研究网络谣言有必要首先对网络與情预 警指标进行分析。我国对于网络與情预警指标 体系的研究开始于2008年, 并在2014年达到顶 峰。戴媛等最早采用AHP法和模糊数学相结合 的办法构建了包含传播扩散、民众关注、内容 敏感性、态度倾向性等四个维度的网络與情预 警指标体系 ${ }^{[5]}$ 。曾润喜在网络與情预警系统的 基础上, 利用层次分析法构建了警源、警兆、 警情三类因素和现象的网络與情突发事件预
警指标体系 ${ }^{[6]}$ 。兰月新基于突发事件网络與情 作用机理和演变规律, 构建了网民反应、突发 事件信息特征、突发事件事态扩散三个维度的 突发事件网络與情安全评估指标体系 ${ }^{[7]}$ 。

\section{1 预警指标构建}

目前, 对网络與情预警指标体系的研究大 多集中在突发性公共事件、旅游危机事件、群 体性事件等正常與情事件上, 对于网络谣言预 警指标体系的研究则较少。戎芯等基于网络谣 言呈现出的独有特征, 构建了扩散广度、内容 热度和态度倾向三个一级指标和 11 个二级指 标的网络谣言影响力因素评价体系, 并选取 “抢盐事件” 进行了实证研究 ${ }^{[8]}$ 。但是该指标 体系中的末级指标大多是定性指标, 不利于进 行定量分析, 无法充分体现突发事件网络谣言 所具有的动态性 ${ }^{[9]}$ 。

在前人研究的基础上, 本文结合现有的网 络與情预警指标特点对网络谣言预警指标进 行了篮选, 构建了网络谣言预警指标体系, 如 图1所示。

\section{2 指标的含义及分析}

本文构建了事件與情热度、网络谣言状 态和网络谣言趋势 3 个一级指标, 每个一级指 标下选取了 3 个具有代表性的二级指标, 共计 9 个末级指标。

\subsection{1 事件興情热度}

\section{(1) 网络搜索量}

当某一敏感突发事件发生时时, 人们迫切 希望知道真相和内情, 但是由于传统媒体报道 具有一定的滞后性, 因此人们会有一个自发的 在网络上寻求信息补足的过程, 因此可以用某 个关键词的网络搜索量来反映人们对某一事 件的关注和兴趣。该指标可以用百度指数来表 示, 百度指数是以网民在百度的搜索量为基础, 科学分析并计算出各个关键词在百度网页搜 索中搜索频次的加权和。

\section{(2) 转发量}

指某条原创微博被其他用户转发的次数, 用户转发该条微博即表示他同意发文者的观 点。微博用户可以分为四种: 发文者、转发者、 评论者和汶览者, 其中汶览者中包括点赞的, 但是由于点赞者也可能参与了转发和评论, 为 


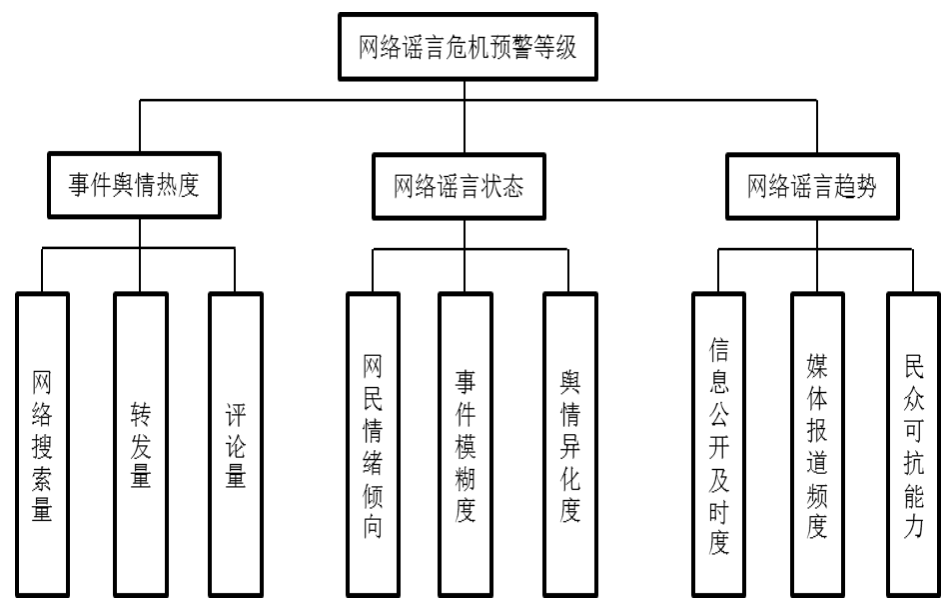

图1 网络谣言预警指标体系

避免数据重复, 在此点赞者不做统计。

\section{（3）评论量}

指某条微博被其他用户评论的次数, 某用 户对发布的原创微博进行评论, 则代表他对其 很关注。如果某条微博被评论的次数较多, 代 表该条微博具有较强的吸引力。

\subsection{2 网络谣言状态}

\section{（1）网民情绪倾向}

指突发事件之后, 网络谣言内容表现出的 情绪倾向，如质疑、害怕等。曾润喜以 “8·12 天津爆炸事故” 为例, 按照 “编号一主题词一 集体情绪” 对谣言文本进行提炼和解读, 并将 其分为 “质疑” “恐慌” 和 “正能量” 三类 ${ }^{[10]}$ 。 其中“质疑”类谣言主要针对政府和主流媒体, 危害程度较高, 赋值为 1 ; “恐慌” 类谣言来源 于造谣者和传谣者的心理特征, 其危害程度次 之, 赋值为 0 ; “正能量” 谣言危害最低, 赋值 为 -1 。该指标由统计时段内各类谣言赋值代 数和来表示。

\section{（2）事件模糊度}

该指标反映事件的模糊程度。美国社会学 家G・W奥尔波特和L - 波斯特曼于1947年提出 谣言影响力公式: 谣言=事件的重要性 $\times$ 事件 的模糊性。该公式表明一件事情和人们的切身 利益相关度越高, 对事件的不确定性程度越大, 那么其滋生谣言的空间和可能性就越大。模糊 程度的测量可以由专家打分法获得, 其取值在 $[0,1]$ 之间。0表示某突发事件刚刚发生时, 原
因, 经过等都不清楚, 存在诸多疑点; 1表示 事情前因后果都十分清楚。

\section{（3）舆情异化度}

正常與情在演化过程中, 会衍生出各种各 样的异化信息, 即网络谣言。网络谣言在流传 过程中会逐步增加或者减少一些信息元素, 由 一个版本演化为多个不同的版本。與情异化度 越高, 政府的防治难度越大。在这里该指标以 不同网络谣言版本数目来表示。

\subsection{3 网络谣言变化趋势}

（1）信息公开及时度

造谣者和辟谣者作为共生对手, 存在于與 论生态系统中 ${ }^{[11]}$ 。敏感性突发事件发生后, 公 众急于知道事情真相，政府和媒体作为权威信 息的发布者, 对于满足公民知情权和消解谣言 起到了至关重要的作用。突发事件后政府和媒 体发布权威消息越及时，谣言产生的概率越低。 该指标用谣言产生后政府或主流媒体发布的 第一条辟谣信息所用平均时间来表示。

\section{（2）媒体报道频度}

该指标用与突发事件相关的微博信息发 布量来表示, 反映了媒体对网络谣言消解的程 度。

\section{（3）民众可抗能力}

传播学者克罗斯对谣言公式进行了进一 步的发展, 提出: 谣言=事件的重要性 $\times$ 事件 的模糊性 $\div$ 公众批判能力。这说明谣言的产生 和传播不仅取决于事件的透明度和重要性, 也 


\section{Risk Analysis and Crisis Response in Big Data Era (RAC-16)}

取决于受众的知识水平, 知识水平越高, 对谣 言的抵抗能力就越强。然而由于个人知识广度 和深度的限制, 不同的人对同一谣言的可抗能 力是不一样的。该指标可由专家打分法获得, 取值区间为 $[0,1]$, 代表面对某一谣言时受众的 平均可抗水平, 0 为没有抗力, 1 为完全能识别 谣言。

\section{3 基于 BP 神经网络的网络谣言危机预警}

\subsection{BP 神经网络预警的优势}

导致网络谣言大规模扩散和传播的因素 有很多, 这些因素之间存在着复杂的非线性关 系, 传统的基于线性关系构建的数学模型不能 很好地模拟导致网络谣言爆发各因素及其之 间的关系。由于 BP 神经网络能学习和存储大 量的输入一输出模式的映射关系, 无需事前揭 示描述这种映射关系的数学方程, 因此, $\mathrm{BP}$ 神经网络非常适合处理内部机制复杂的、非线 性的, 具有全局性问题特征的实际问题, 被广 泛应用于模式识别、图像处理和各种自然灾害 预警中。此外, BP 神经网络具有的高度自适 应学习能力和较强的容错能力使得 BP 神经网 络成为网络谣言危机预警的一种理想选择。

\section{2 预警模型构建}

BP 神经网络一种层次结构的人工神经网 络, 包含输入层、隐含层和输出层, 层与层之 间采用全互连的方式，而同一层的神经元之间 不存在连接, 其中隐含层可以包含多层。虽然 增加隐含层可以提高网络精度, 降低误差, 但 是也会使得网络复杂化, 降低网络的学习速率。 因此本文采用单隐层的三层 BP 神经网络, 由 输入层输入处理过的末级指标数据, 输出层输 出相应的预警级别。

\section{3 输入数据的归一化处理}

无论是定性还是定量指标都有正向指标 和负向指标之分, 为了使各指标在整个系统中 具有可比性, 因此必须对各指标进行归一化的 标准化处理, 将它们转化为 $[0,1]$ 上的无量纲化 指标值。定性、定量指标数据归一化处理方式 在下面详述。

\subsection{1 正向指标处理}

正向指标是指值越大越好的指标, 在本 文的网络谣言危机预警中, 是指指标值越大越 安全, 危机等级越小。其无量纲化处理以最小 值为基准, 公式如下:

$$
x=\frac{x_{i}-x_{\min }}{x_{\max }-x_{\min }}
$$

其中, $x$ 为量化后的指标值, $x_{i}$ 为指标的 实际值, $x_{\text {min }}$ 为指标的最小值, $x_{\max }$ 为指标 的最大值。

\subsection{2 负向指标处理}

负向指标是指值越小越好的指标, 在本 文中, 该指标越小越安全, 危机等级越小。其 无量纲化处理以最大值为基准, 公式如下:

$$
x=\frac{x_{\max }-x_{i}}{x_{\max }-x_{\min }}
$$

\section{4 输出节点及隐含节点的选择}

《国家突发公共事件总体应急预案》将突 发公共事件预警等级划分为四级: IV 级 (一般)、 III级 (较重)、II 级 (严重)、I 级 (特别严重), 依次用蓝色、黄色、橙色和红色表示。基于此, 本文将网络谣言危机预警等级划分为四级: 安 全、轻警、中警、重警, 输出状态为 1000 、 0100、0010、0001, 分别对应四个预警等级。 隐含层节点数 $\mathrm{N}$ 常常采用公式（3）来确 定:

$$
N=\sqrt{m+n}+\alpha
$$

其中 $m$ 为输入层节点数, $n$ 为输出层节点数, $\alpha$ 为 1 到 10 之间的常数。

\section{5 训练参数的设置}

本文利用 MATLAB R 2007b 软件自带的 $B P$ 神经网络工具箱来建立网络模型。隐含层 和输出层传递函数为 $\mathrm{S}$ 型函数 “logsig”, 训练 函数采用 “traingdx”, 最大训练次数为 1000 , 训练目标误差为 0.01 , 网络学习速率为 0.05 , 动量系数为 0.9 。

\section{4 仿真实验}

\section{1 数据来源}

选取 2015 年 8 月 12 日 23:30 左右发生的 “天津爆炸” 事故作为研究对象。数据主要来 
Risk Analysis and Crisis Response in Big Data Era (RAC-16)

源于百度搜索引擎提供的百度指数和新浪微 博提供的数据。

\section{2 数据样本与处理}

2015 年 8 月 12 日 23:30 分左右, 位于天 津市滨海新区天津港的瑞海公司危险品仓库 发生火灾爆炸事故, 造成重大人员伤亡和巨大 直接经济损失。此次爆炸事故 12 日 23:30 左 右发生, 13 日引发大规模网络與情, 到 23 日 事故引发的网络與情逐渐平息。此次突发事件 在网络上掀起與论风暴的同时, 也衍生出了各 种各样的网络谣言, 据统计此次事件前后共产 生了 27 个不同版本的网络谣言。这些网络谣 言的产生模式不同于一般的网络谣言, 没有经 过漫长的酝酿期而是在爆炸事件后第二天集 中爆发, 并且谣言从产生到消解时间极短, 非 常符合突发事件网络谣言的相关特征。因此选 取该事件作为研究对象对于突发事件中的网 络谣言危机预警研究具有很强的代表意义。

关于此次爆炸事件的网络谣言从13日晚 开始产生, 到 16 日晚基本全部消解完毕。由 于网络谣言爆发时间相对集中, 因此本文以 6 个小时为单位, 将 12 日晚 18:00 到 16 日晚 18:00划分为 16 个时间段进行相关数据的采集 和统计, 并对原始数据进行归一化处理, 这样 9 个末级指标数据就有了可比性。事件的时间 段划分如表 1 所示:

表 1 时间段划分

\begin{tabular}{|c|c|c|c|}
\hline 序号 & 时间段 & 序号 & 时间段 \\
\hline 1 & 12 日 18: $00-24: 00$ & 9 & 14日 18: $00-24: 00$ \\
\hline 2 & 13日 00: 00-06: 00 & 10 & 15日00: 00-06: 00 \\
\hline 3 & 13日 06: $00-12: 00$ & 11 & 15日 06: $00-12: 00$ \\
\hline 4 & 13日 12: $00-18: 00$ & 12 & 15日 12: $00-18: 00$ \\
\hline 5 & 13日 18: $00-24: 00$ & 13 & 15日 18: $00-24: 00$ \\
\hline 6 & 14日 00: 00-06: 00 & 14 & 16日 00: $00-06: 00$ \\
\hline 7 & 14日 06: $00-12: 00$ & 15 & 16日 06: $00-12: 00$ \\
\hline 8 & 14 日 12: $00-18: 00$ & 16 & 16日 12: $00-18: 00$ \\
\hline
\end{tabular}

对网络谣言危机预警指标体系中的 9 个 末级指标的性质进行分析, 分析结果如表 2 所 示。

一级指标事件與情热度中的三个二级指 标数据可以通过百度指数和新浪微博 (以 “新 浪天津” 微博账号在各相应时间段发布的原创 微博中转发量和评论量最高的发文作为数据 获取来源) 直接查询得到。网络谣言状态中的

\begin{tabular}{ccc}
\multicolumn{4}{c}{ 表 2 指标性质 } \\
\hline 变量 & 内容 & 性质 \\
\hline$X_{1}$ & 网络搜索量 & 定量, 负向指标 \\
$X_{2}$ & 转发量 & 定量, 负向指标 \\
$X_{3}$ & 评论量 & 定量, 负向指标 \\
$X_{4}$ & 谣言情绪倾向 & 定量, 负向指标 \\
$X_{5}$ & 事件模糊度 & 定性, 正向指标 \\
$X_{6}$ & 與情异化度 & 定量, 负向指标 \\
$X_{7}$ & 信息公开及时度 & 定量, 负向指标 \\
$X_{8}$ & 媒体报道频度 & 定量, 正向指标 \\
$X_{9}$ & 民众可抗能力 & 定性, 正向指标 \\
\hline
\end{tabular}

网民情绪倾向指标经过统计获得, 事件模糊度 由专家打分法获得, 與情异化度通过统计不同 谣言版本数目获得。网络谣言趋势中的媒体报 道频度指标通过统计“新浪天津” 各时间段 (统 计时间从 8 月 12 日 23:30 到 8 月 16 日 18:00) 的发文数量得到, 信息公开及时度指标通过网 络搜索各类谣言产生后第一条官方辟谣信息 发出所用平均时间得到。搜集到的原始数据经 过去重和去无关联处理之后如表 3 所示。 在得出原始数据后, 还要根据各指标的性质对 原始数据作归一化处理, 处理方法为利用公式

（1）和公式（2）将每一项指标值都归一化到 区间 $[0,1]$ 上, 处理后的数据如表 4 所示。

\section{3 实验验证}

\subsubsection{BP 神经网络模型的结构设计}

采用单隐层的三层 $\mathrm{BP}$ 神经网络。在输入 节点数 $m$ 为 9 , 输出节点数 $n$ 为 4 的情况下, 根据隐含层节点计算公式 (3), 将 $\alpha$ 从 1 到 10 逐个计算, 发现当 $\alpha$ 等于 4 时, 网络性能 最佳, 此时隐含层节点数为 8 。

\subsection{2 基于 Matlab 的 BP 神经网络模型训练}

选用表 5 中经过标准化处理后的前 15 个 指标数据作为模型的训练样本, 最后一个数据 作为模型的检验样本。它们对应的期望输出如 表 5 和表 6 所示。

\subsection{3 实验结果分析}

把第 16 组数据实际输出的转换结果与表 7 的期望输出值进行比较, 看是否一致, 一致 这说明该模型是有效的, 否则说明神经网络构 建的不合理, 需要进一步改进。最后的实验结 
Risk Analysis and Crisis Response in Big Data Era (RAC-16)

表 3 原始数据

\begin{tabular}{|c|c|c|c|c|c|c|c|c|c|}
\hline 时间 & $\mathrm{X}_{1}$ & $\mathrm{X}_{2}$ & $\mathrm{X}_{3}$ & $\mathrm{X}_{4}$ & $\mathrm{X}_{5}$ & $\mathrm{X}_{6}$ & $\mathrm{X}_{7}$ & $\mathrm{X}_{8}$ & $\mathrm{X}_{9}$ \\
\hline 时间1 & 3972 & 0 & 0 & 0 & 0 & 0 & 0 & 0 & 1 \\
\hline 时间2 & 968720 & 10920 & 1052 & 4 & 0.1 & 8 & 12.36 & 6 & 0.2 \\
\hline 时间3 & 1227890 & 12730 & 1616 & -1 & 0.1 & 5 & 10.2 & 11 & 0.4 \\
\hline 时间4 & 1489654 & 13756 & 1055 & -1 & 0.4 & 6 & 11.07 & 8 & 0.3 \\
\hline 时间5 & 1098742 & 4379 & 3334 & -1 & 0.6 & 2 & 11.67 & 7 & 0.7 \\
\hline 时间6 & 803816 & 1177 & 652 & 0 & 0.6 & 0 & 0 & 1 & 1 \\
\hline 时间7 & 1184873 & 2176 & 1011 & 0 & 0.6 & 1 & 1.33 & 5 & 0.5 \\
\hline 时间8 & 1026539 & 967 & 542 & -1 & 0.6 & 1 & 2.67 & 4 & 0.5 \\
\hline 时间9 & 903671 & 496 & 645 & 0 & 0.7 & 0 & 0 & 6 & 1 \\
\hline 时间10 & 534028 & 327 & 146 & 0 & 0.7 & 0 & 0 & 1 & 1 \\
\hline 时间11 & 1267964 & 728 & 193 & 0 & 0.7 & 1 & 6.58 & 2 & 0.5 \\
\hline 时间12 & 1375290 & 196 & 197 & -1 & 0.7 & 2 & 6.5 & 7 & 0.7 \\
\hline 时间13 & 509087 & 918 & 857 & 0 & 0.8 & 1 & 7.34 & 3 & 0.5 \\
\hline 时间14 & 758939 & 55 & 123 & 0 & 0.8 & 0 & 0 & 1 & 1 \\
\hline 时间15 & 1071256 & 454 & 236 & 0 & 0.8 & 0 & 0 & 3 & 1 \\
\hline 时间16 & 1190873 & 920 & 1963 & 0 & 0.8 & 0 & 0 & 6 & 1 \\
\hline
\end{tabular}

表 4 标准化后数据

\begin{tabular}{|c|c|c|c|c|c|c|c|c|c|}
\hline 间 & $x_{1}$ & $x_{2}$ & $x_{3}$ & $X_{4}$ & $X_{5}$ & $X_{6}$ & $x_{7}$ & $X_{8}$ & $X_{9}$ \\
\hline 时间1 & 1 & 1 & 1 & 0.8 & 0 & 1 & 1 & 0 & 1 \\
\hline 时间2 & 0.351 & 0.206 & 0.684 & 0 & 0.125 & 0 & 0 & 0.545 & 0 \\
\hline 时间3 & 0.176 & 0.075 & 0.515 & 1 & 0.125 & 0.375 & 0.175 & 1 & 0.25 \\
\hline 时间4 & 0 & 0 & 0.684 & 1 & 0.5 & 0.25 & 0.104 & 0.727 & 0.125 \\
\hline 时间 5 & 0.263 & 0.682 & 0 & 1 & 0.75 & 0.75 & 0.056 & 0.636 & 0.625 \\
\hline 时间6 & 0.462 & 0.914 & 0.804 & 0.8 & 0.75 & 1 & 1 & 0.091 & 1 \\
\hline 时间7 & 0.205 & 0.842 & 0.697 & 0.8 & 0.75 & 0.875 & 0.892 & 0.455 & 0.375 \\
\hline 时间8 & 0.312 & 0.930 & 0.837 & 1 & 0.75 & 0.875 & 0.784 & 0.364 & 0.375 \\
\hline 时间 9 & 0.394 & 0.964 & 0.807 & 0.8 & 0.875 & 1 & 1 & 0.546 & 1 \\
\hline 时间10 & 0.643 & 0.976 & 0.956 & 0.8 & 0.875 & 1 & 1 & 0.091 & 1 \\
\hline 时间11 & 0.149 & 0.947 & 0.942 & 0.8 & 0.875 & 0.875 & 0.468 & 0.182 & 0.375 \\
\hline 时间 12 & 0.077 & 0.986 & 0.941 & 1 & 0.875 & 0.75 & 0.474 & 0.636 & 0.625 \\
\hline 时间 13 & 0.660 & 0.933 & 0.743 & 0.8 & 1 & 0.875 & 0.406 & 0.273 & 0.375 \\
\hline 时间 14 & 0.492 & 0.996 & 0.963 & 0.8 & 1 & 1 & 1 & 0.091 & 1 \\
\hline 时间 15 & 0.282 & 0.967 & 0.929 & 0.8 & 1 & 1 & 1 & 0.273 & 1 \\
\hline 时间16 & 0.201 & 0.933 & 0.411 & 0.8 & 1 & 1 & 1 & 0.545 & 1 \\
\hline
\end{tabular}


Risk Analysis and Crisis Response in Big Data Era (RAC-16)

表 5 训练样本期望输出

\begin{tabular}{|c|c|c|c|}
\hline $\begin{array}{c}\text { 时间序 } \\
\text { 号 }\end{array}$ & 期望输出 & $\begin{array}{c}\text { 危机等 } \\
\text { 级 }\end{array}$ & $\begin{array}{c}\text { 预警信 } \\
\text { 号 }\end{array}$ \\
\hline 时间 1 & 1000 & 安全 & 蓝色 \\
\hline 时间 2 & 0100 & 轻警 & 黄色 \\
\hline 时间 3 & 0001 & 重警 & 红色 \\
\hline 时间 4 & 0001 & 重警 & 红色 \\
\hline 时间 5 & 0001 & 重警 & 红色 \\
\hline 时间 6 & 0010 & 中警 & 橙色 \\
\hline 时间 7 & 0010 & 中警 & 橙色 \\
\hline 时间 8 & 0010 & 中警 & 橙色 \\
\hline 时间 9 & 0010 & 中警 & 橙色 \\
\hline 时间 10 & 0100 & 轻警 & 黄色 \\
\hline 时间 11 & 0100 & 轻警 & 黄色 \\
\hline 时间 12 & 0100 & 轻警 & 黄色 \\
\hline 时间 13 & 0100 & 轻警 & 黄色 \\
\hline 时间 14 & 1000 & 安全 & 蓝色 \\
\hline 时间 15 & 1000 & 安全 & 蓝色 \\
\hline
\end{tabular}

表 6 测试样本的期望输出

\begin{tabular}{|c|c|c|c|}
\hline 时间序号 & 期望输出 & 危机等级 & 预警信号 \\
\hline 时间 16 & 1000 & 安全 & 蓝色 \\
\hline
\end{tabular}

果表明, 实际输出的转换结果与期望输出是一 致的, 发出了蓝色预警信号 (1000), 表明此 时网络谣言已全部消解。仿真实验表明, 本文 构建的网络谣言预警指标体系是合理的, 基于 BP 神经网络的突发事件网络谣言危机预警模 型是有效的。

\section{5 结论}

政府和媒体作为权威信息的主要发布者, 对于突发事件网络谣言的预防和消解至关重 要。由于突发事件处置信息发布的滞后性, 不 可能堵塞住所有谣言, 这就需要政府部门能对 突发事件网络谣言进行有效监测, 才能及时辟 谣, 最大程度降低谣言带来的负面影响。本文 通过建立突发事件网络谣言预警指标体系, 构 建了基于 BP 神经网络的网络谣言危机预警模 型, 最后结合案例进行仿真实验验证了该模型 的可行性。解决如何识别、规避、权衡风险的 问题, 是人们得以正常生活的基本保障 ${ }^{[12]}$, 希 望该研究能够提升政府对突发事件网络谣言 的监测能力和预警能力。接下来的研究方向就 是进一步完善网络谣言预警指标体系, 减少乃
至消除定性指标, 提高数据采集的及时性和有 效性, 最终实现计算机实时监测及自动预警。

\section{参考文献}

[1]中国互联网络信息中心.第37次中国互联网 络发展状况统计报告. http://cnnic.cn/gywn/ xwZX/rdxw/2015/201601/t20160122_53283.

htm, 2016-01-22.

[2] 张鹏.基于 HAYASHI 数量化理论的网络谣 言分类应对策略. 情报杂志，2016(1): 110-115.

[3] 敖炀.多理论视角下网络谣言的成因与对 策浅析. 品牌, 2012(1): 13-15.

[4] 黄建. 论网络谣言的协同防控. 中南大学学 报，2013(3):105-108.

[5] 戴媛.我国网络與情安全评估指标体系研 究.北京化工大学硕士学位论文, 2008 .

[6] 曾润喜.网络舆情突发事件预警指标体系 构建.情报理论与实践，2010(1):77-80。

[7] 兰月新.突发事件网络與情安全评估指标 体系构建. 图书情报工作, 2011(1):317 -319.

[8] 戎荵, 兰月新等.网络谣言影响力因素评价 体系及策略研究. 现代情报, 2014 , 34(10):25-30.

[9] 曹蓉.基于全样本分析的网络與情指标体 系研究综述. 情报杂志，2015(5):154-158.

[10] 曾润喜, 魏冯. 政媒共治: 灾难事件中网 络造谣与辟谣的信息行为研究. 电子政务, 2016(5):25-34.

[11]姜景, 李丁, 刘怡君.基于竞争模型的微博 谣言信息与辟谣信息传播机理研究. 数学的 实践与认识, 2015(1):182-191.

[12] Huang C F. Internet of Intelligences in Risk Analysis for Online Services. The Journal of Risk Analysis and Crisis Response, 2011(2):110-117. 